\title{
On relation between nebular morphology and infrared photometric properties
}

\author{
Slawomir K. Górny and Natasza Siódmiak
}

N. Copernicus Astronomical Center, Toruń, Poland

\begin{abstract}
We report on the ongoing study of the infrared properties of different types of planetary nebulae based on the data from recent photometric and spectroscopic infrared surveys. We present our first results concerning the relation between nebular morphology and infrared properties using the observations from the AKARI mission.
\end{abstract}

Keywords. planetary nebulae: general

We have compiled a list 2643 Galactic planetary nebulae (PNe) based on the catalogues of Acker et al. (1992), Kerber et al. (2003), Parker et al. (2006) and Miszalski et al. (2008). For the two most recent catalogues we have used the original coordinates supplied by the authors and the coordinates from CDS Simbad database for the remaining objects. We have removed possible misclassified PNe from the list using the information from the same source. The search within the AKARI database resulted in identifying $754 \mathrm{PNe}$ registered by the IRC camera and 930 in the far infrared by FIS instrument.

We have analysed the statistics of the PNe detected by AKARI at different wavelengths as the function of the optical diameter finding that the highest ratio of detections is for the smallest nebulae with diameters $<5^{\prime \prime}$ reaching $69 \%$ in band F18. On the other hand, only a small number of PNe larger than 60 " have been detected reaching about $10 \%$ at F65 and longer wavelengths.

In a similar way statistics for PNe grouped by their morphology have been investigated. The data on morphological types of PNe have been taken from Górny (2001) and combined with the morphological information included in Parker et al. (2006) and Miszalski et al. (2006) conforming them for this study to a simplified scheme of morphological classes as presented in Fig. 1. It consists of four main categories of PNe describing their general shape: stellar-like $(S)$, elliptical $(E)$, bipolar $(B)$ and irregular $(I)$. (Objects of other morphology, e.g. spherically round, point-symmetric or of multi-axis geometry have not been considered).

We have found the highest rate of AKARI detections among $S$ PNe that strongly peaks with $55 \%$ of detections in band F18. In the same band $33 \%$ of $I$ PNe have been registered by AKARI although their rate of detections is more uniformly distributed as is also the case of $B$ and $E$ PNe.

In Fig. 2 we show positions of the PNe of different morphology in two AKARI colourcolour planes using only the data with the best quality flag. The typical locations of the four morphological types clearly differ. In particular, in left panel of Fig. 2 it can be seen that $S$ and $B$ PNe have different F65/F18 colours. This could be interpreted as the result of the difference in evolutionary stage since the stellar-like PNe are naturally the smallest among nebulae and it could be expected that the nebula needs to expand before its more complicated morphology will be recognised. As the dust temperature decreases with time the F65/F18 ratio would increase (see Phillips \& Marquez-Lugo 2011) explaining the observed larger values for $B$ than $S$ PNe. On the other hand, in the 


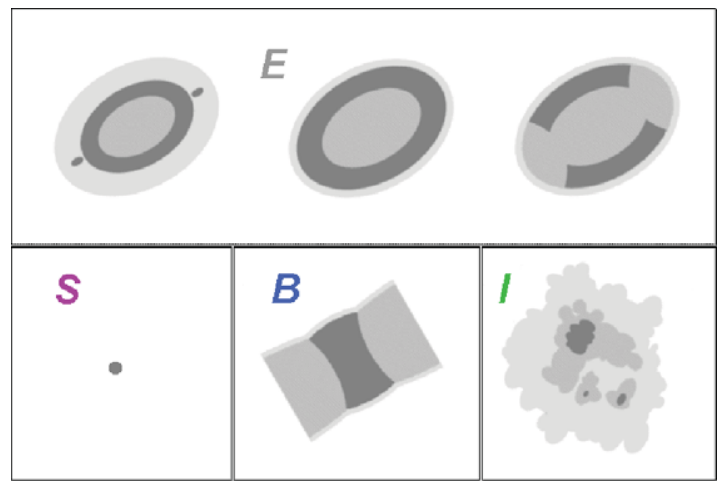

Figure 1. Sketches of the adopted simplified morphological classification: $E$ - elliptical; $S$ - stellar; $B$ - bipolar; $I$ - irregular
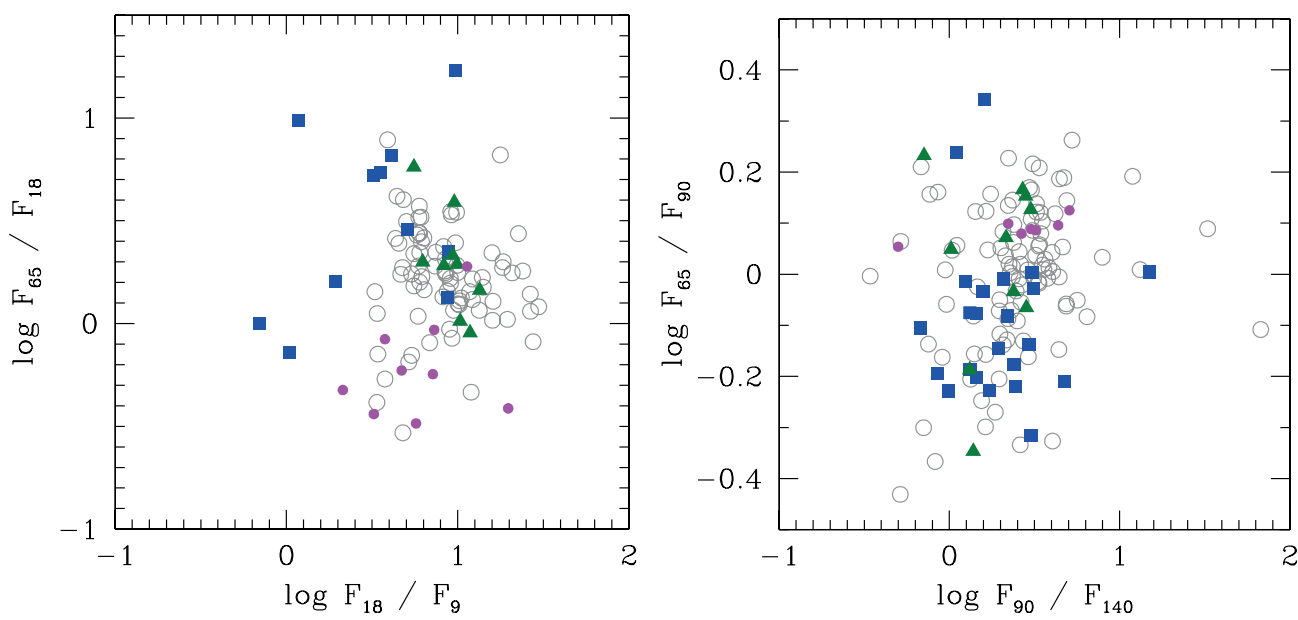

Figure 2. Positions of elliptical (circles), bipolar (squares), irregular (triangles) and stellar $\mathrm{PNe}$ (dots) in two AKARI colour-colour planes.

right panel of Fig. 2 the $S$ type objects are not among the PNe with the largest F65/F90 ratio contradicting such simple explanation.

The positions of objects with $I$ morphology also differ from $B$ PNe. For example, they seem to have different F18/F9 colours. This indicates that PNe of different morphological types can be distinguished not only by their apparent shape but also molecular and dust composition related to their earlier evolution on AGB.

\section{Acknowledgements}

This work was supported from grant N N203 511838 of the Science and High Education Ministry of Poland.

\section{References}

Acker, A., Ochsenbein, F., Stenholm, B., et al. 1992, Strasbourg-ESO Catalogue

Górny, S. K. 2007, Ap\&SSS, 275, 67

Kerber, F., Mignani, R. P., Guglielmetti, F., \& Wicenec, A. 2003, A\&A, 408, 1029

Miszalski, B., Parker, Q., Acker, A., et al. 2008, MNRAS, 384, 525

Parker, Q., Acker, A., Frew, D. J., et al. 2006, MNRAS, 373, 79

Phillips, J. P. \& Marquez-Lugo, R. A. 2011, RMxAA, 47, 83 